\title{
CAPTURA DE DIÓXIDO DE CARBONO EM ALTAS TEMPERATURAS POR MEIO DA REAÇÃO DE CARBONATAÇÃO DO ORTOSSILICATO DE LÍTIO
}

\author{
S. M. AMORIM ${ }^{1}$, M. D. DOMENICO ${ }^{1}$, T. L. P. DANTAS ${ }^{2}$, H. J. JOSÉ ${ }^{1}$ e R. F. P. M. MOREIRA ${ }^{1}$ \\ ${ }^{1}$ Universidade Federal de Santa Catarina, Departamento de Engenharia Química e Engenharia de \\ Alimentos \\ ${ }^{2}$ Universidade Federal do Paraná, Departamento de Engenharia Química \\ E-mail para contato: amorim_sm@yahoo.com.br
}

\begin{abstract}
RESUMO - Uma das mais promissoras técnicas de captura de $\mathrm{CO}_{2}$ em altas temperaturas consiste na sua separação por meio da reação de carbonatação com um sólido inorgânico. Neste trabalho, foi avaliada a cinética da captura de $\mathrm{CO}_{2}$ do ortossilicato de lítio $\left(\mathrm{Li}_{4} \mathrm{SiO}_{4}\right)$ à pressão atmosférica na faixa de temperatura de 500 a $900{ }^{\circ} \mathrm{C}$. O sólido $(97,5 \%$ de pureza) foi caracterizado pelas análises de estrutura porosa, difração de raios $\mathrm{X}$, microscopia eletrônica de varredura e análise térmica. A análise de parâmetros termodinâmicos indicou que a captura pelo $\mathrm{Li}_{4} \mathrm{SiO}_{4}$ é fortemente dependente da temperatura, sendo o sólido capaz de adsorver $36,7 \% \mathrm{CO}_{2}$ em massa na temperatura de equilíbrio $\left(723{ }^{\circ} \mathrm{C}\right)$. De acordo com o ensaio não-isotérmico, a reação de carbonatação ocorre na faixa de $500-735{ }^{\circ} \mathrm{C}$, sendo que em temperaturas acima de $735^{\circ} \mathrm{C}$ ocorre a reação de descarbonatação. Os ensaios isotérmicos mostraram que o aumento da temperatura favorece a reação, e, em altas temperaturas, é possível capturar $35 \% \mathrm{CO}_{2} \mathrm{em}$ massa, valor muito próximo à capacidade teórica máxima.
\end{abstract}

\section{INTRODUÇÃO}

A reforma a vapor do metano e a gaseificação de carvão mineral, realizados em altas temperaturas $\left(700-900{ }^{\circ} \mathrm{C}\right)$, têm como produto o dióxido de carbono e a sua retirada do meio reacional, além de aumentar a concentração dos produtos gasosos desejados, ainda melhora a eficiência dos processos (Halabi et al., 2011).

Uma das mais promissoras técnicas de captura de dióxido de carbono consiste na sua separação por meio da reação reversível com um adsorvente. Sólidos como o carvão ativado e as zeólitas, por exemplo, têm sido largamente estudados, porém, as baixas temperaturas de equilíbrio de adsorção $\left(25-150{ }^{\circ} \mathrm{C}\right)$ limitam a sua aplicação. $\mathrm{O}$ óxido de cálcio $(\mathrm{CaO})$, por outro lado, é capaz de capturar uma considerável quantidade de $\mathrm{CO}_{2}$ em altas temperaturas (600-700 ${ }^{\circ} \mathrm{C}$ ), mas apresenta baixa estabilidade em repetidos ciclos de carbonatação/descarbonatação e necessita de alta energia para sua regeneração $\left(950^{\circ} \mathrm{C}\right)$ (Yin et al., 2010).

As cerâmicas a base de lítio tais como o óxido de lítio $\left(\mathrm{Li}_{2} \mathrm{O}\right)$, o zirconato de lítio $\left(\mathrm{Li}_{2} \mathrm{ZrO}_{3}\right)$, o silicato de lítio $\left(\mathrm{Li}_{4} \mathrm{SiO}_{4}\right)$, o aluminato de lítio $\left(\mathrm{Li}_{5} \mathrm{AlO}_{4}\right)$, a ferrita de lítio $\left(\mathrm{LiFeO}_{2}\right)$ e 


\section{9 a 22 de outubro de 2014 \\ Florianópolis/SC}

o titanato de lítio $\left(\mathrm{Li}_{4} \mathrm{TiO}_{4}\right)$ têm sido estudadas para a captura de $\mathrm{CO}_{2}$ (Ortiz-Landeros et al., 2012). $\mathrm{O} \mathrm{Li} \mathrm{Li}_{2} \mathrm{O}$ apresenta a maior capacidade de captura por unidade de massa (valor teórico de $147,3 \%$ ), porém, sua alta reatividade e corrosão limitam o seu uso. $\mathrm{O} \mathrm{Li}_{5} \mathrm{AlO}_{4}$ também se destaca pela alta capacidade (valor teórico de 70,0 \% em massa) e ampla faixa de operação $\left(200-700{ }^{\circ} \mathrm{C}\right)$, contudo, devido ao efeito de sinterização, responsável por reduzir a área superficial, ocorre a diminuição da reatividade do sólido (Ávalos-Rendón et al., 2009). A alta capacidade do $\mathrm{Li}_{2} \mathrm{SiO}_{3}$ (valor teórico de $48,9 \%$ em massa) não o torna competitivo para captura em altas temperaturas, pois a temperatura de equilíbrio da reação com $\mathrm{CO}_{2}$ é de $260{ }^{\circ} \mathrm{C}$ (Nakagawa et al., 2003). $\mathrm{O}$ $\mathrm{Li}_{4} \mathrm{TiO}_{4}$, o $\mathrm{LiFeO}_{2}$ e o $\mathrm{Li}_{2} \mathrm{ZrO}_{3}$ possuem as menores capacidades teóricas de captura com valores iguais a 31,5\%;23,2 \% e 28,7 \% em massa, respectivamente. Nakagawa et al. (2003) reportaram que a temperatura de equilíbrio da reação do $\mathrm{LiFeO}_{2}$ é relativamente baixa $\left(510^{\circ} \mathrm{C}\right)$ se comparada com a de outras cerâmicas de lítio como o $\mathrm{Li}_{2} \mathrm{ZrO}_{3}\left(715^{\circ} \mathrm{C}\right)$ e o $\mathrm{Li}_{4} \mathrm{SiO}_{4}\left(720{ }^{\circ} \mathrm{C}\right)$.

Dentre os compostos a base de lítio, o ortossilicato de lítio se destaca como um material promissor nesta aplicação, pois, apresenta uma boa capacidade de captura (valor teórico de 36,7 $\%$ em massa) de acordo com a reação de carbonatação $\mathrm{Li}_{4} \mathrm{SiO}_{4(s)}+\mathrm{CO}_{2(g)} \leftrightarrow \mathrm{Li}_{2} \mathrm{SiO}_{3(\mathrm{~s})}+$ $\mathrm{Li}_{2} \mathrm{CO}_{3(s)}$, rápida cinética de carbonatação/descarbonatação, excelente estabilidade após vários ciclos e boas propriedades mecânicas (Seggiani et al., 2011; Wang et al., 2011).

Muitos autores têm estudado métodos de síntese para produzir este sólido adsorvente (Pfeiffer et al. 1998; Chang et al., 2001; Bretado et al., 2005; Wang et al., 2011), mas nenhum trabalho foi publicado explorando as alternativas já existentes no mercado. Neste trabalho, foi realizada a caracterização do ortossilicato de lítio obtido comercialmente, com o objetivo de analisar as propriedades físico-químicas e a cinética de adsorção do $\mathrm{CO}_{2}$.

\section{SEÇÃO EXPERIMENTAL}

\subsection{Materiais}

$\mathrm{O}$ ortossilicato de lítio $\left(\mathrm{Li}_{4} \mathrm{SiO}_{4}\right)$ utilizado neste estudo foi produzido pela Chemetall e apresenta-se na forma de um pó cristalino com uma pureza de aproximadamente 97,5\%. Os gases utilizados nas análises termogravimétricas foram fornecidos pela White Martins Ltda: nitrogênio (99,996 vol. \% de pureza) e dióxido de carbono industrial.

\subsection{Métodos}

A área superficial do sólido foi calculada pelo método BET e a isoterma de adsorção/dessorção (a qual relaciona o volume de $\mathrm{N}_{2}$ adsorvido em equilíbrio com sua pressão parcial na fase gasosa) foi obtida utilizando o equipamento Nova 2200e (Quantachrome). O tamanho e a morfologia das partículas foram determinados na análise de microscopia eletrônica de varredura (MEV) utilizando um microscópio JEOL JSM-6390LV. As fases cristalinas presentes no sólido foram encontradas por meio da análise de difração de raios X (DRX), a qual foi conduzida em um Difratômetro X'Pert (Philips) com scan de 0,038 /s e radiação de $\mathrm{Cu} \mathrm{K \alpha}$. A análise de decomposição térmica do sólido foi realizada em um analisador termogravimétrico 
DTG-60 (Shimadzu) com a finalidade de identificar a presença de impurezas na estrutura do $\mathrm{Li}_{4} \mathrm{SiO}_{4}$. A análise consistiu em um aquecimento com taxa de $5{ }^{\circ} \mathrm{C} /$ min desde a temperatura ambiente até $950{ }^{\circ} \mathrm{C}$, em atmosfera de $\mathrm{N}_{2}$.

Os experimentos de captura de $\mathrm{CO}_{2}$ foram realizados no analisador termogravimétrico DTG-60 (Shimadzu) em duas etapas. Primeiramente, foi conduzida uma análise térmica dinâmica, na qual o sólido foi aquecido a $10{ }^{\circ} \mathrm{C} / \mathrm{min}$ em uma atmosfera com $100 \%$ de $\mathrm{CO}_{2}$, desde a temperatura ambiente até $1000{ }^{\circ} \mathrm{C}$. O fluxo de alimentação de $\mathrm{CO}_{2}$ no equipamento foi mantido constante e igual a $200 \mathrm{~mL} / \mathrm{min}$. Na sequência, os experimentos isotérmicos de carbonatação foram conduzidos numa faixa de 500 à $730^{\circ} \mathrm{C}$ utilizando um fluxo de $200 \mathrm{~mL} / \mathrm{min}$ de $\mathrm{CO}_{2}$. Antes de cada ensaio, o sólido foi submetido a um pré-tratamento para eliminar substâncias indesejáveis em sua superfície. O pré-tratamento consistiu em um aquecimento com taxa de $5{ }^{\circ} \mathrm{C} / \mathrm{min}$ desde a temperatura ambiente até $750{ }^{\circ} \mathrm{C}$ em atmosfera de $\mathrm{N}_{2}$.

As reações de equilíbrio termodinâmico entre o ortossilicato de lítio e dióxido de carbono em diferentes temperaturas foram determinadas teoricamente utilizando o programa FactSage 6.3 (FACT - Facility for the Analysis of Chemical Thermodynamics, versão disponibilizada online).

\section{RESULTADOS E DISCUSSÕES}

\subsection{Caracterização}

A análise textural do ortossilicato de lítio gerou uma isoterma de adsorção/dessorção de $\mathrm{N}_{2}$ do tipo II (Figura 1) típica de adsorvente não-poroso ou macroporoso. Nesse tipo de isoterma, a formação da monocamada em baixas pressões parciais tem como processo dominante a adsorção das moléculas. Já em altas pressões parciais a adsorção passa a ocorrer em multicamadas e a espessura do adsorbato aumenta progressivamente até que a pressão de condensação seja atingida (Leofanti et al., 1998).

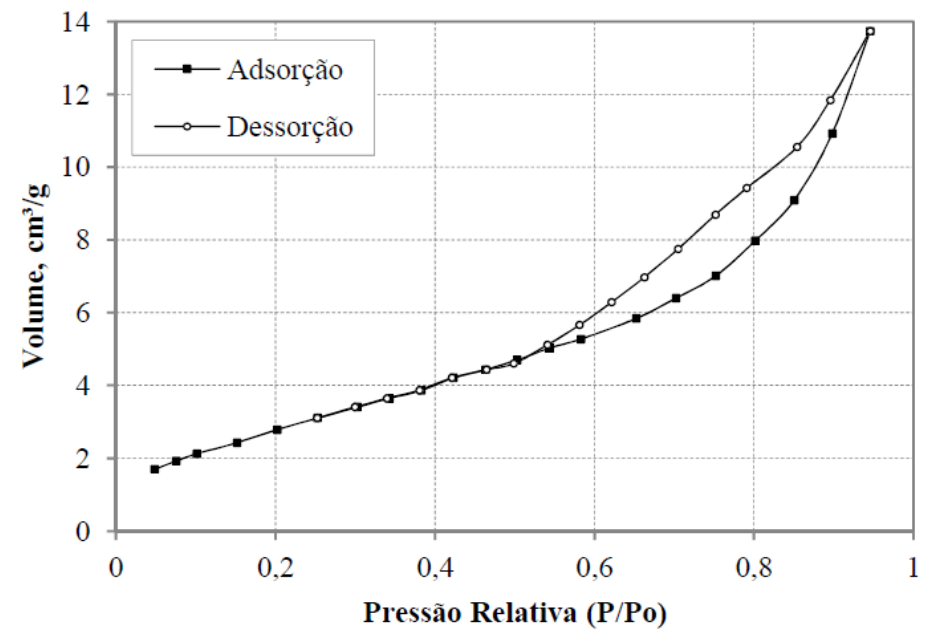

Figura 1 - Isoterma de adsorção/dessorção de $\mathrm{N}_{2}$ a $77 \mathrm{~K}$ do $\mathrm{Li}_{4} \mathrm{SiO}_{4}$. 
A área superficial específica determinada pelo método BET apresentou um valor de aproximadamente $11 \mathrm{~m}^{2} / \mathrm{g}$, o que concorda com a classificação do sólido como não-poroso. Uma pequena área superficial pode ser um fator limitante na carbonatação, pois o $\mathrm{CO}_{2}$ pode não ter acesso a toda área ativa do sólido, havendo a necessidade de difusão do gás através da camada de produto ou difusão dos íons de lítio e oxigênio até a superfície para que a reação prossiga.

De acordo com a análise de MEV mostrada na Figura (a), o $\mathrm{Li}_{4} \mathrm{SiO}_{4}$ possui densas partículas poliédricas irregulares e em alguns pontos aglomeradas com diferentes tamanhos, que variam até aproximadamente $350 \mu \mathrm{m}$. A aproximação na Figura 2 (b) mostra a superfície de uma partícula com aspecto liso, confirmando a baixa área superficial encontrada pela análise textural.
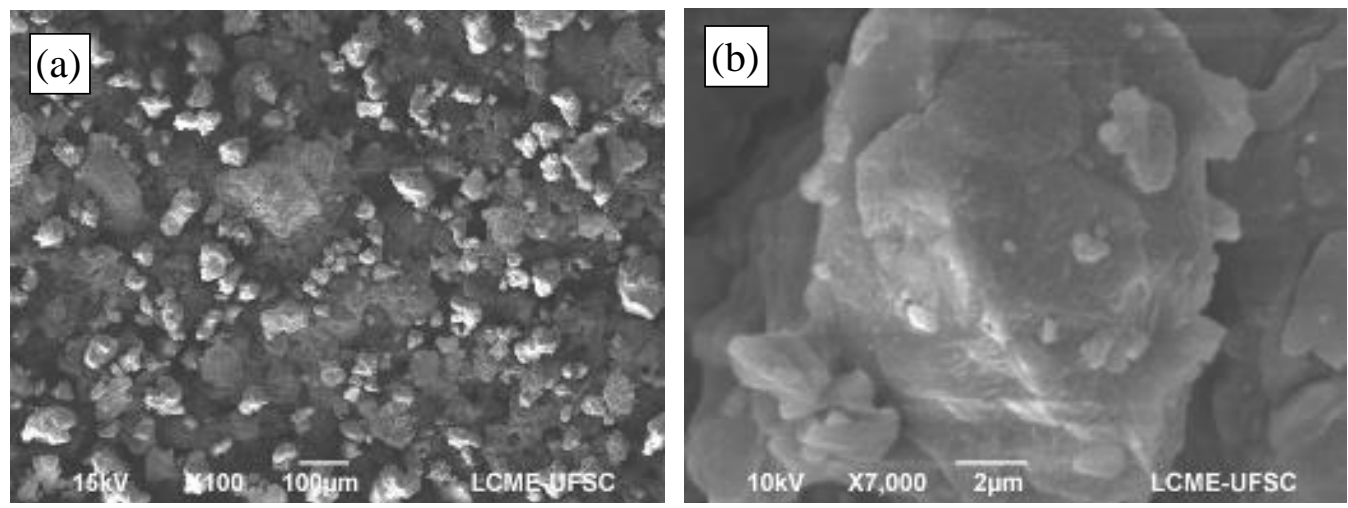

Figura 2 - Análises de MEV do $\mathrm{Li}_{4} \mathrm{SiO}_{4}$ com aproximações de 100x (a) e 7000x (b).

O difratograma de raios $\mathrm{X}$ do $\mathrm{Li}_{4} \mathrm{SiO}_{4}$ ortossilicato de lítio (Figura 3) apresentou picos característicos das fases cristalinas do material $\left(\mathrm{Li}_{4} \mathrm{SiO}_{4}\right.$ - JCPDS 37-1472), metassilicato de lítio $\left(\mathrm{Li}_{2} \mathrm{SiO}_{3}\right.$ - JCPDS 83-1517), carbonato de lítio $\left(\mathrm{Li}_{2} \mathrm{CO}_{3}\right.$ - JCPDS 83-1454), hidróxido de lítio hidratado (LiOH.H $\mathrm{H}_{2} \mathrm{O}$ - JCPDS 76-1073) e dióxido de silício ( $\mathrm{SiO}_{2}$ - JCPDS 82-1568).

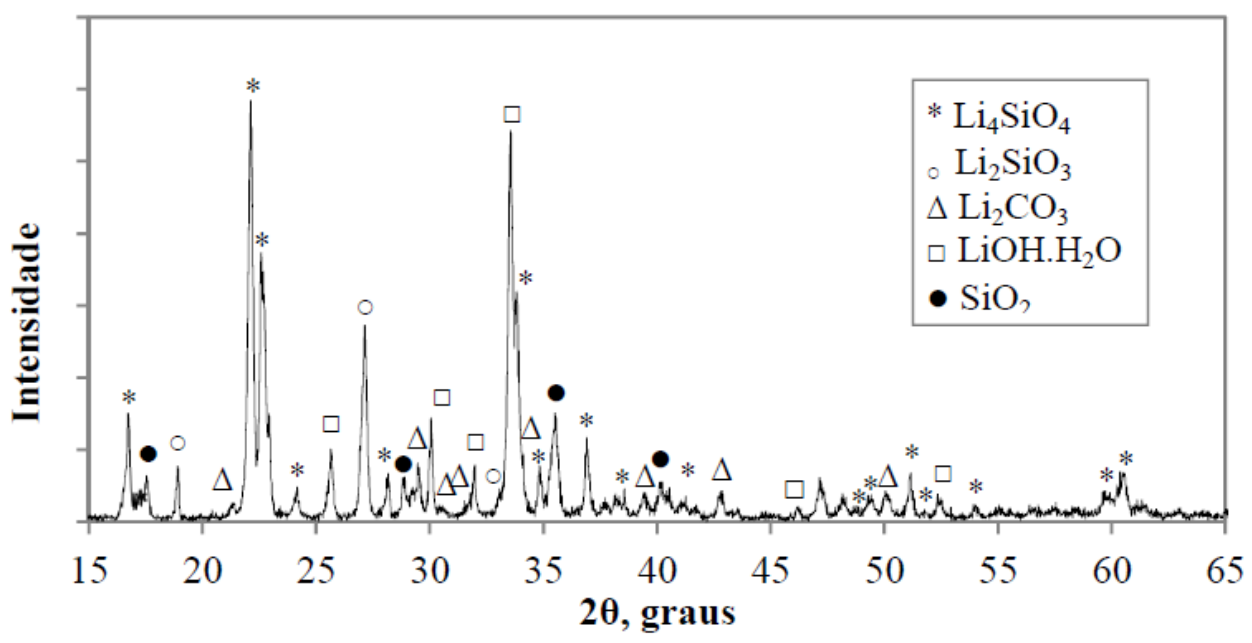

Figura 3 - Difratograma de raios $\mathrm{X}$ do $\mathrm{Li}_{4} \mathrm{SiO}_{4}$. 
A presença dos compostos cristalinos $\mathrm{LiOH} . \mathrm{H}_{2} \mathrm{O}, \mathrm{Li}_{2} \mathrm{CO}_{3}$ e $\mathrm{SiO}_{2}$ pode ser explicada pela reação do $\mathrm{Li}_{4} \mathrm{SiO}_{4}$ com água na forma de vapor em temperatura ambiente conforme estudado por Ortiz-Landeros et al. (2011) e/ou com dióxido de carbono conforme estudado por Kato et al. (2005). As reações com água e dióxido de carbono ocorrem em temperatura ambiente e de forma lenta, portanto, a sua ocorrência pode ser atribuída ao tempo que a amostra ficou armazenada antes da sua utilização.

$\mathrm{Na}$ análise de decomposição térmica do ortossilicato de lítio, mostrada na Figura 4, foram identificadas quatro etapas onde ocorre a perda de massa. As etapas 1 e 2 consistem na eliminação da água presente na superfície do sólido conforme reportado por Nakagawa et al. (2003) e Cruz et al. (2006). A etapa 3 é atribuída ao processo de desidroxilação do $\mathrm{Li}_{4} \mathrm{SiO}_{4}$ (Pfeiffer et al., 1998; Chang et al., 2001). A presença de hidróxido de lítio foi detectada na análise de DRX para o ortossilicato de lítio comercial (Figura 3), o que concorda com o resultado da análise de decomposição térmica aqui mostrada. A descarbonatação é a quarta e última etapa na análise de decomposição térmica e a presença do $\mathrm{CO}_{2}$ no material também confirmou o resultado obtido na análise de DRX na qual foram identificados os produtos cristalinos $\mathrm{Li}_{2} \mathrm{CO}_{3} \mathrm{e}$ $\mathrm{SiO}_{2}$.

A perda total de massa resultante do aquecimento do ortossilicato de lítio entre as temperaturas de $28{ }^{\circ} \mathrm{C}$ e $950{ }^{\circ} \mathrm{C}$ foi igual a $12 \%$, indicando a presença de impurezas e tornando necessária a realização de um pré-tratamento antes dos experimentos de carbonatação.

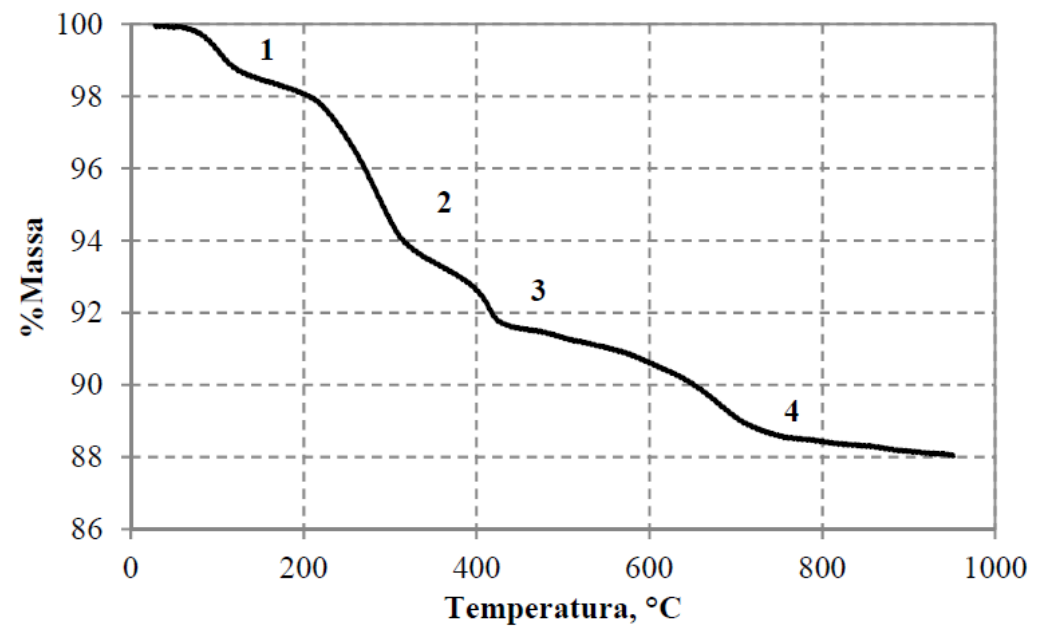

Figura 4 - Análise térmica dinâmica do $\mathrm{Li}_{4} \mathrm{SiO}_{4}$ em atmosfera de nitrogênio.

\subsection{Equilíbrio Termodinâmico}

As reações de equilíbrio termodinâmico entre o ortossilicato de lítio e dióxido de carbono obtidas pelo programa FactSage 6.3 são apresentadas na Tabela 1. Verificou-se que o adsorvente reage com $\mathrm{CO}_{2}$ gerando diferentes produtos, dependendo da faixa de temperatura da reação. Ainda, foi observado que a relação estequiométrica $\mathrm{CO}_{2}: \mathrm{Li}_{4} \mathrm{SiO}_{4}$ diminui com o aumento da 
temperatura, o que é um aspecto desfavorável do ponto de vista da captura de $\mathrm{CO}_{2}$. Por outro lado, foi verificado que as reações de carbonatação são muito lentas em temperaturas baixas (25$\left.262^{\circ} \mathrm{C}\right)$.

Tabela 1 - Reações entre $\mathrm{Li}_{4} \mathrm{SiO}_{4}$ e $\mathrm{CO}_{2}$ a diferentes temperaturas

\begin{tabular}{cc}
\hline Faixa Temperatura $\left({ }^{\circ} \mathrm{C}\right)$ & Reação \\
\hline $25-228$ & $\mathrm{Li}_{4} \mathrm{SiO}_{4}+2 \mathrm{CO}_{2} \leftrightarrow 3 \mathrm{Li}_{2} \mathrm{CO}_{3}+\mathrm{SiO}_{2}$ \\
$229-262$ & $2 \mathrm{Li}_{4} \mathrm{SiO}_{4}+3 \mathrm{CO}_{2} \leftrightarrow 3 \mathrm{Li}_{2} \mathrm{CO}_{3}+\mathrm{Li}_{2} \mathrm{Si}_{2} \mathrm{O}_{5}$ \\
$262-723$ & $\mathrm{Li}_{4} \mathrm{SiO}_{4}+\mathrm{CO}_{2} \leftrightarrow \mathrm{Li}_{2} \mathrm{CO}_{3}+\mathrm{Li}_{2} \mathrm{SiO}_{3}$ \\
$724-1000$ & Etapa de descarbonatação \\
\hline
\end{tabular}

\subsection{Cinética da Reação de Carbonatação}

De acordo com a análise térmica dinâmica do ortossilicato de lítio, mostrada na Figura 5, a faixa de temperatura na qual ocorreu a carbonatação do $\mathrm{Li}_{4} \mathrm{SiO}_{4}$ está entre 500 e $735{ }^{\circ} \mathrm{C}$. Ainda, verificou-se que nesta faixa, a variação da massa foi de aproximadamente 33\%, valor próximo à capacidade teórica máxima calculada para este sólido (36,7 \% em massa).

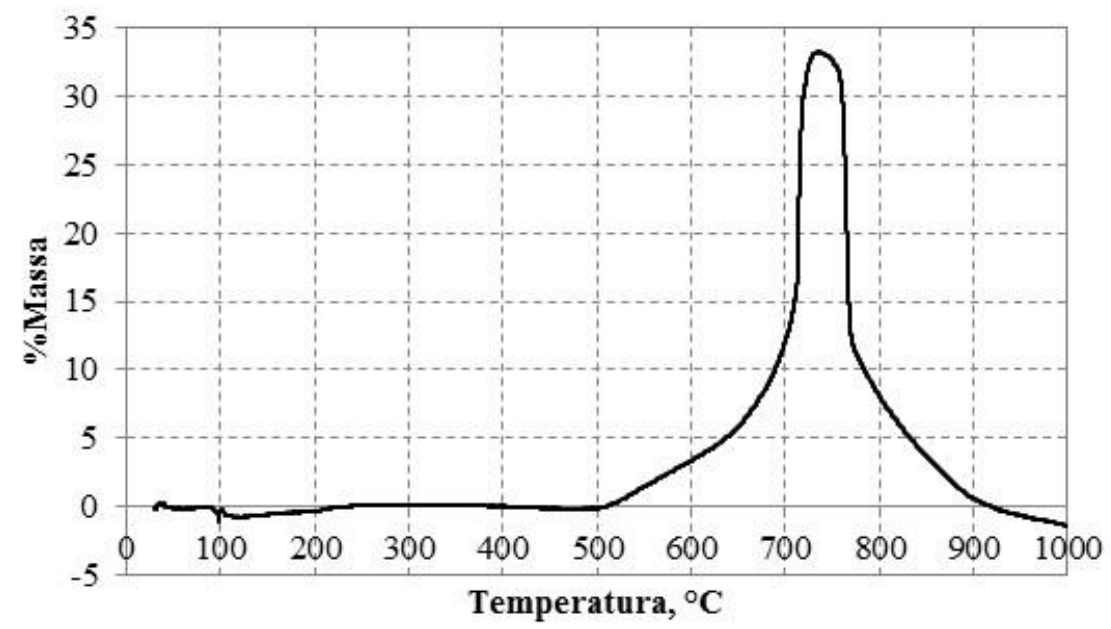

Figura 5 - Análise térmica dinâmica do $\mathrm{Li}_{4} \mathrm{SiO}_{4}$ em atmosfera de $\mathrm{CO}_{2}$.

O fenômeno de descarbonatação do $\mathrm{Li}_{4} \mathrm{SiO}_{4}$, por sua vez, ocorreu a partir de $735{ }^{\circ} \mathrm{C}$, temperatura próxima aquela encontrada na análise teórica dos produtos gerados pelo programa FactSage 6.3. A massa final do sólido foi menor do que a inicial, o que significa que em temperaturas acima de $950{ }^{\circ} \mathrm{C}$ ocorreu um processo de decomposição, dado pela equação $\mathrm{Li}_{4} \mathrm{SiO}_{4(s)} \leftrightarrow \mathrm{Li}_{2} \mathrm{SiO}_{3(s)}+\mathrm{Li}_{2} \mathrm{O}_{(\mathrm{g})}(\mathrm{Cruz}$ et al., 2006).

$\mathrm{O}$ mecanismo para captura de $\mathrm{CO}_{2}$ nos compostos de lítio parece ocorrer em duas etapas. Primeiramente, ocorre a reação do $\mathrm{CO}_{2}$ na superfície da partícula até a completa formação da 
camada de produtos, composta principalmente por carbonato de lítio $\left(\mathrm{Li}_{2} \mathrm{CO}_{3}\right)$. Na segunda etapa, a reação passa a ser controlada por processos difusivos, seja pela difusão do lítio nos produtos da reação ou difusão do $\mathrm{CO}_{2}$ pela camada de $\mathrm{Li}_{2} \mathrm{CO}_{3}$ (Ortiz-Landeros et al. 2012). De acordo com as isotermas mostradas na Figura 6, a cinética da reação de carbonatação foi muito rápida no início, devido à reação do $\mathrm{CO}_{2}$ na superfície exposta do $\mathrm{Li}_{4} \mathrm{SiO}_{4}$. À medida que a reação avança, a velocidade diminuiu devido a limitações difusivas. Contudo, com o aumento da temperatura, a carbonatação ocorreu mais rapidamente e a limitação pelos processos difusivos foi praticamente eliminada. Segundo Huang e Daugherty (1987), esse comportamento decorre da sinterização do carbonato de lítio em altas temperaturas (acima de $700{ }^{\circ} \mathrm{C}$ ) e provoca o aumento na área superficial das partículas em contato com a atmosfera gasosa. Na temperatura de $730{ }^{\circ} \mathrm{C}$, o $\mathrm{Li}_{2} \mathrm{CO}_{3}$ encontra-se fundido, facilitando a difusão através desse produto.

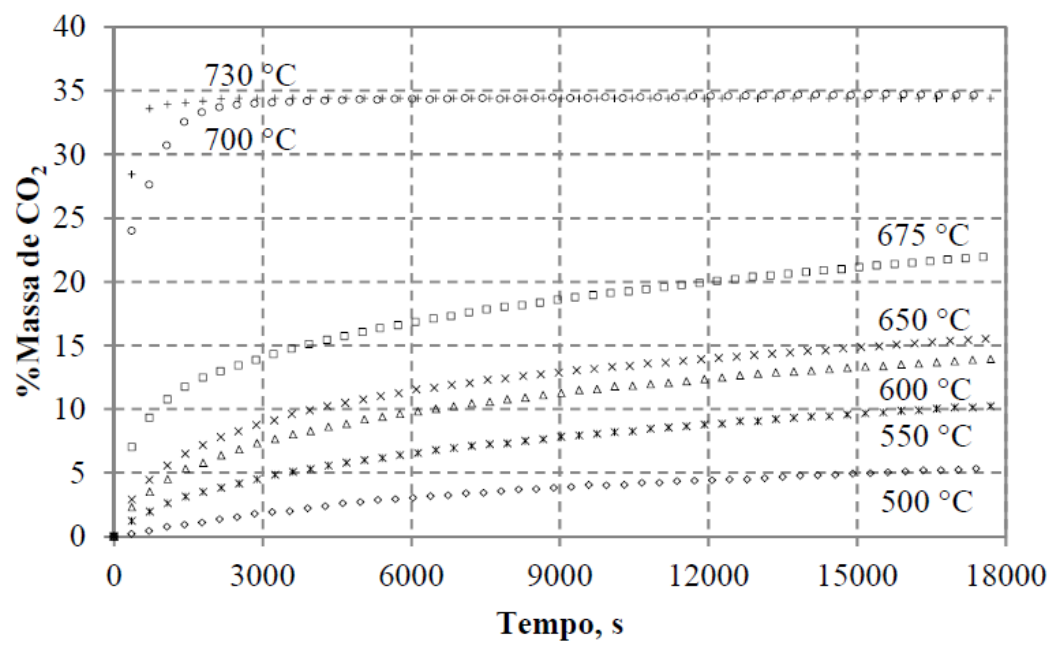

Figura 6 - Cinética da reação de carbonatação do $\mathrm{Li}_{4} \mathrm{SiO}_{4}$ em diferentes temperaturas.

\section{CONCLUSÕES}

Neste trabalho estudou-se a aplicação de uma cerâmica de lítio $\left(\mathrm{Li}_{4} \mathrm{SiO}_{4}\right)$ comercial na captura de dióxido de carbono em altas temperaturas. A ampla faixa de temperatura de carbonatação $\left(500-735^{\circ} \mathrm{C}\right)$, a possibilidade de regeneração em temperaturas mais baixas $\left(735^{\circ} \mathrm{C}\right)$ se comparado com outros sólidos estudados para o mesmo fim e sua a alta capacidade de captura de $\mathrm{CO}_{2}\left(35 \%\right.$ em massa de $\left.\mathrm{CO}_{2}\right)$ tornam esse sólido comercial competitivo para aplicação na captura de $\mathrm{CO}_{2}$ em altas temperaturas.

\section{REFERÊNCIAS}

ÁVALOS-RENDÓN, T.; CASA-MADRID, J.; PFEIFFER, H.. Thermochemical capture of carbon dioxide on lithium aluminates $\left(\mathrm{LiAlO}_{2}\right.$ and $\left.\mathrm{Li}_{5} \mathrm{AlO}_{4}\right)$ : A new option for the $\mathrm{CO}_{2}$ absorption. J. Phys. Chem. A, v. 113, n. 25, p. 6919-6923, 2009. 


\section{9 a 22 de outubro de 2014 \\ Florianópolis/SC}

BRETADO, M. E.; VELDERRAIN, V. G.; GUTIÉRREZ, D. L.; COLLINS-MARTÍNEZ, V.; ORTIZ, A. L.. A new synthesis route to $\mathrm{Li}_{4} \mathrm{SiO}_{4}$ as $\mathrm{CO}_{2}$ catalytic/sorbent. Catal. Today, v. 107-108, p. 863- 867, 2005.

CHANG, C. C.; WANG, C. C.; KUMTA, P. N.. Chemical synthesis and characterization of lithium orthosilicate $\left(\mathrm{Li}_{4} \mathrm{SiO}_{4}\right)$. Mater. Design, v. 22, n. 7, p. 617-623, 2001.

CRUZ, D.; BULBULIAN, S.; LIMA, E.; PFEIFFER, H.. Kinetic analysis of the thermal stability of lithium silicates $\left(\mathrm{Li}_{4} \mathrm{SiO}_{4}\right.$ and $\left.\mathrm{Li}_{2} \mathrm{SiO}_{3}\right)$. J. Solid State Chem., v. 179, n. 3, p. 909-916, 2006.

HALABI, M. H.; CROON, M. H. J. M. de; SCHAAF, J. van der; COBDEN, P. D.; SCHOUTEN, J. C.. Reactor modeling of sorption-enhanced autothermal reforming of methane part I: Performace study of hydrotalcite and lithium zirconate-based processes. Chem. Eng. J., v. 168 , n. 2, p. 1-11, 2011.

HUANG, J.; DAUGHERTY, K. E.. Catalytic effect of alkali carbonates on the calcination of calcium carbonate. Thermochim. Acta, v. 115, p. 57-62, 1987.

KATO, M.; NAKAGAWA, K.; ESSAKI, K.; MAEZAWA, Y.; TAKEDA, S.; KOGO, R.; HAGIWARA, Y.. Novel $\mathrm{CO}_{2}$ absorbents using lithium-containing oxide. Int. J. of Appl. Ceram. Technol., v. 2, n. 6, p. 467-475, 2005.

LEOFANTI, G.; PADOVAN, M.; TOZZOLA, G.; VENTURELLI, B.. Surface area and pore texture of catalysts. Catal. Today, v. 41, n. 1-3, p. 207-219, 1998.

NAKAGAWA, K.; KATO, M.; YOSHIKAWA, S.; ESSAKI, K.; UEMOTO, H.. A novel $\mathrm{CO}_{2}$ absorbent using lithium-containing oxides, in Proceedings of the 2nd Annual Conference on Carbon Sequestration, Alexandria VA, mai., 2003.

ORTIZ-LANDEROS, J.; MARTÍNEZ-DLCRUZ, L.; GÓMES-YÁÑES, C.; PFEIFFER, H.. Towards understanding the thermoanalysis of water sortpion on lithium orthosilicate $\left(\mathrm{Li}_{4} \mathrm{SiO}_{4}\right)$. Thermochim. Acta, v. 515, n. 2, p. 73-78, 2011.

ORTIZ-LANDEROS, J.; ÁVALOS-RÉNDON, T. L.; GÓMEZ-YÁÑES, C.; PFEIFFER, H. Analysis and perspectives concerning $\mathrm{CO}_{2}$ chemisorption on lithium ceramics using thermal analysis. J. Therm. Anal. Calorim., v. 102, n.2, p. 647-655, 2012.

PFEIFFER, H.; BOSCH, P.; BULBULIAN, S.. Synthesis of lithium silicates. J. Nucl. Mater., v. 257, n. 3, p. 309-317, 1998.

SEGGIANI, M.; PUCCINI, M.; VITOLO, S.. High- temperature and low concentration $\mathrm{CO}_{2}$ sorption on $\mathrm{Li}_{4} \mathrm{SiO}_{4}$ based sorbents: Study of the used silica and doping method effects. Int. J. Greenh. Gas Con., v. 5, n. 4, p. 741-748, 2011.

YIN, X.; SONG, M.; ZHANG, Q.; YU, J.. High-temperature $\mathrm{CO}_{2}$ Capture on $\mathrm{Li}_{6} \mathrm{Zr}_{2} \mathrm{O}_{7}$ : Experimental and modeling studies. Ind. Eng. Chem. Res., v. 49, n. 14, p. 6593-6598, 2010.

WANG, K.; GUO, X.; ZHAO, P.; WANG, F.; ZHENG, C.. High temperature capture of $\mathrm{CO}_{2}$ on lithium-based sorbents from rice husk ash. J. Hazard. Mater., v. 189, n. 1-2, p. 1-7, 2011. 